\title{
Recoding processes in recognition: Some effects of presentation rate*
}

\author{
CHARLES CLIFTON, JR. \\ University of Massachusetts, Amherst, Massachusetts 01002 \\ DONNA CRUSE \\ Oregon State University, Corvallis, Oregon 97331 \\ and \\ KARL D. GUTSCHERA \\ University of Massachusetts, Amherst, Massachusetts 01002
}

\begin{abstract}
Character classification time was studied in a situation in which Ss sometimes had to decide whether a probe item was a translation of a remembered item according to a previously learned translation scheme. When translation between probe and remembered items was necessary, the rate at which the to-be-remembered items had been presented affected the form of the memory-scanning functions. This result seemed to contradict Cruse and Clifton's (1973) hypothesis that Ss translated all remembered items into the form of the probe after the probe was presented. An alternative model claimed that, when time permits, Ss translate to-be-remembered items into their alternate form at the time of presentation and scan the resulting translated items at a relatively slow rate if the probe matches them in form. If $\mathrm{Ss}$ are unable to translate the to-be-remembered items when they are presented, they resort to the strategy of translating the probe item into the form in which the remembered items had been presented.
\end{abstract}

People rarely store information in the form in which they perceive it. Rather, they recode it into a form that is more easily retained and retrieved. A good deal has been learned about such recoding processes (e.g., Miller, 1956; Mandler, 1967; Sachs, 1967; Bower, 1970; Bransford \& Franks, 1971; Melton \& Martin, 1972).

The complementary process, that of decoding information from memory, has received less intensive investigation (but see Bower, 1970 and Prytulak, 1971). Often, information that has been stored in memory in one form must be decoded into another form to meet the demands of a task. Swanson, Johnsen, and Briggs (1972) and Cruse and Clifton (1973) have reported some results that might bear on such a decoding process. using Sternberg's (1966) character classification task. Sternberg's task consists of presenting $S$ with a set of items to remember, followed by a single probe item. The $S$ indicates whether the probe item was a member of the preceding memory set. Sternberg $(1966,1969)$ found that mean recognition time increases as a linear function of the number of items in memory and that positive and negative response functions have equal slopes. He concluded that a serial and exhaustive scan of the memory items was employed in memory retrieval. Thus, $R T=a+b s$, where $s$ is the number of items in the memory set, $b$ is the time to compare the probe item with a single item in the set, and $a$ is a constant including the time to perceive and encode the probe, to make the response, etc.

*Supported in part by Grant MH-23939-01 to the first author and NSF Grant GU 4041 to the Department of Psychology, University of Massachusetts. The authors express their appreciation to Eric Brewer for conducting Experiment II and to Jerome Myers for a critical reading of the manuscript.
Cruse and Clifton (1973) modified the original task so that recoding processes could be determined from the RT functions. Prior to the decision-making task, Ss learned a translation scheme that provided them with two alternate codes for items to be remembered. On half of the trials, the form of the probe item differed from the form of the items in the memory set, requiring recoding at some point. Specifically, Ss were trained in the scheme $A=1, B=2, \ldots H=8$, enabling them to recode letters as digits and vice versa. A set of one to four letters or one to four digits was presented on each trial, followed by a single letter or digit probe. The $S$ was to make a "yes" response if either the probe or its translation was a member of the memory set.

The functions relating $\mathrm{RT}$ to the size of the memorized set revealed two clear findings. First, the slope was $150 \%$ greater when the memory and probe forms differed than when the probes were the same. Second, the zero intercepts did not differ significantly as a function of recoding requirements. These results indicated that $S s$ did not translate the probe item to match the remembered items in form. Such a process would have increased the zero intercepts, not the slopes, of the RT functions.

Cruse and Clifton suggested two hypotheses that could account for their data. Their first hypothesis, the "list translation" hypothesis (also suggested by Swanson et al, 1972) claimed that Ss store the memorized set only in its presented form (or in a simpler form if one exists, e.g., in the case of binary to octal translation). When a probe that differs from the remembered items in form is presented, Ss translate the items in the remembered set to match the form of the probe. Thus, when translation is required, the following equation 
would hold: $R T=a+(b+t) s$, where $t$ is the duration of a translation of a remembered item into the form of the probe. Under this hypothesis, the difference in slope between the conditions in which translation is and is not required is a measure of how long it takes to decode a single item from memory into the form of the probe.

Their second hypothesis, "dual code," claimed that S translates each memory item as it is presented and stores both the presented and the translated forms. However, only one form (typically the presented form) is rehearsed, and this form is held in active memory where it may be scanned rapidly. The other, nonrehearsed form is stored in a less rapidly scanned inactive memory. Cruse and Clifton expressed misgivings about this hypothesis, because it apparently makes unreasonable demands on memory span. It seemed unlikely that Ss could store up to four octal digits plus four triples of binary digits, as required in Cruse and Clifton's Experiment 2, in which Ss' use of the binary-octal translation scheme was studied.

The present experiments are designed to test these two hypotheses by varying the rate of presentation of the memory set and the delay before the probe. Under the list translation hypothesis, there is no clear reason to expect rate of presentation to have an effect, as long as it is slow enough to allow Ss to perceive and encode the presented items. However, under the dual code hypothesis, it should be possible to find a presentation rate that is slow enough to allow the memory items to be encoded but too fast to allow them to be translated into the alternate form prior to the probe presentation. At this fast presentation rate, Ss could not decide about a probe item differing in form from the presented items by scanning some translated representations in inactive memory. Rather, $\mathrm{S}$ would have to resort to some other strategy, which would very likely have an effect on the RT functions. For instance, one strategy would be to translate the probe item into the form of the remembered items, thus increasing the zero intercept but not the slope of the RT functions, relative to a condition in which no recoding was required.

\section{EXPERIMENT I}

Method

\section{Subjects}

Eight experienced Ss served for one practice and six experimental sessions of $1 \mathrm{~h}$ each. The $S$ s were instructed to respond as quickly and as accurately as possible and were paid according to a formula which increased pay as a linear function of speed and decreased pay 15 cents for each error.

\section{Equipment}

The stimuli were presented on a Burroughs Alphanumeric Nixie tube (B-5871), controlled by a PDP 8/I computer. The display tube was approximately $2.5 \mathrm{~m}$ in front of the two $\mathrm{Ss}$, who were run at the same time, and subtended a visual angle of $0.4 \mathrm{deg}$ in the horizontal plane. Each S's console had two vertical response levers and a central ready light/button which was illuminated when an error was made and when a trial block was about to begin. The light remained on until pressed by $\mathrm{S}$. Both Ss wore headphones that presented continuous white noise and warning tones.

\section{Procedure}

Twenty practice trials, followed by nine blocks of 32 trials each, were presented in each session. Each trial block represented a single presentation rate condition (fast, medium, slow), with each condition occurring on three blocks within a session. The ordering of the rate variable was counterbalanced over blocks and was explained to Ss prior to the first block.

To begin each trial, one to four digits or one to four letters were presented successively at a fast, medium, or slow rate $(400$, 1000 , or $1600 \mathrm{msec} / \mathrm{item}$, respectively). The display tube was blanked during the last $100 \mathrm{msec}$ of each item's presentation time. The items making up the positive set were chosen at random from a set of eight (A-H and 1-8) and varied unpredictably from trial to trial, as did the number of items in the set.

The 100-msec blank interval following the last item in the target set was followed by another blank interval of 0,1600 , or $2400 \mathrm{msec}$ for the fast, medium, and slow rate conditions, respectively. A $100-\mathrm{msec}$ warning tone then occurred and, $300 \mathrm{msec}$ later, a probe item (either a digit or a letter) appeared on the display tube. If either the probe item or its translation was contained in the target set, $\mathrm{S}$ was to pull the right (positive) lever with his right index finger; otherwise, $S$ was to pull the left (negative) lever. Half of the probes required translation and an orthogonal half of the probes required a positive response. Serial position of positive probes and identity of negative probes were chosen randomly. If $S$ made an erroneous response, he had to press an illuminated center button. Two seconds after all responses were made, the next trial began. Each of the 32 conditions defined by the combination of set size (1-4), set form (digits or letters), probe form, and response (positive or negative) occurred once in each trial block.

\section{Results and Discussion}

The mean RT of correct responses (excluding the $0.9 \%$ of all responses which had latencies less than 300 or greater than $1400 \mathrm{msec}$ ) was calculated for each $S$ on each block of 2 days for each experimental condition. The digit set, digit probe (D-D) and letter set, letter probe (L-L) conditions were averaged to produce a "no translation" (NT) condition, and the D-L and L-D conditions were averaged to produce a "translation" (T) condition. (Statistical analyses were performed on the data prior to such averaging.) The means of these means, together with percentages of false alarm and miss errors, are presented in Fig. 1. The 95\% confidence interval on these mean RTs, estimated from the error term of the analysis of variance described later, was $\pm 19.85 \mathrm{msec} /$ item. The error rates are seen to be fairly high, exceeding $10 \%$ in some conditions. However, error rates in Experiment II (with conditions comparable to the fast condition of Experiment I) were much lower, while the RT functions were nearly identical.

The RT functions for the NT conditions appear to be quite linear and parametrically similar at all presentation rates, supporting the claim that $S s$ in the fast rate 
Fig. 1. RT as a function of set size, Experiment $\mathrm{I}$, with $\%$ miss and false alarm $\mathrm{er}$ rors. Dashed lines, and stippled bars, correspond to $\mathrm{T}$ conditions; solid lines and bars to NT conditions.

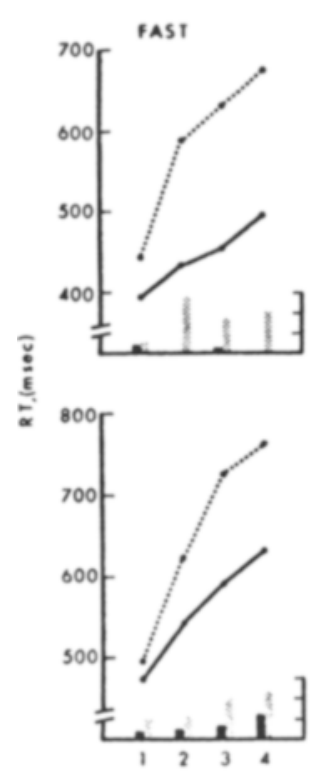

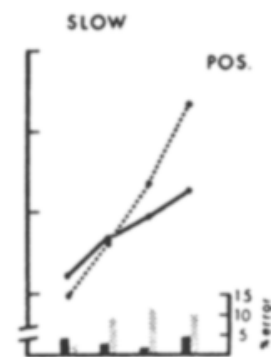

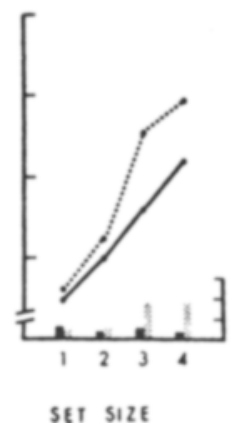

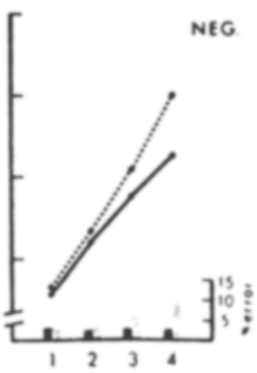

Table 1

Parameters of Straight Lines Fitted (Least Squares) to Mean RTs: Experiment I

\begin{tabular}{|c|c|c|c|c|}
\hline \multirow[b]{3}{*}{ Parameter } & \multicolumn{4}{|c|}{ Condition } \\
\hline & \multicolumn{2}{|c|}{ No Translation } & \multicolumn{2}{|c|}{ Translation } \\
\hline & $\begin{array}{c}\text { Posi- } \\
\text { tive }\end{array}$ & $\begin{array}{c}\text { Nega- } \\
\text { tive }\end{array}$ & $\begin{array}{l}\text { Posi- } \\
\text { tive }\end{array}$ & $\begin{array}{c}\text { Nega- } \\
\text { tive }\end{array}$ \\
\hline & \multicolumn{4}{|c|}{ Fast } \\
\hline Slope & 32.7 & 52.1 & 73.8 & 91.4 \\
\hline Zero Intercept & 363 & 429 & 401 & 424 \\
\hline \multirow[t]{2}{*}{ Percent Variance } & 99 & 99 & 90 & 95 \\
\hline & \multicolumn{4}{|c|}{ Medium } \\
\hline Slope & 33.6 & 58.3 & 71.3 & 80.3 \\
\hline Zero Intercept & 384 & 390 & 323 & 377 \\
\hline \multirow[t]{2}{*}{ Percent Variance } & 97 & 99 & 99 & 99 \\
\hline & \multicolumn{4}{|c|}{ Slow } \\
\hline Slope & 32.9 & 55.7 & 76.7 & 79.3 \\
\hline Zero Intercept & 395 & 405 & 314 & 377 \\
\hline Percent Variance & 99 & 99 & 99 & 99 \\
\hline
\end{tabular}

Note-Percent variance is the squared product-moment correlation coefficient between obtained RTs and RTs estimated by the fitted straight line.

Table 2

Significant $(p<.01)$ Effects, Main Analysis: Experiment I

\begin{tabular}{llr}
\hline \multicolumn{1}{c}{ Effect } & df & \multicolumn{1}{c}{ F } \\
\hline Set Size $(\mathrm{N})$ & 3,21 & 33.72 \\
Response $(\mathrm{R})$ & 1,7 & 34.29 \\
Rate $(\mathrm{T})$ & 2,14 & 21.61 \\
$\mathrm{~N}$ by R & 3,21 & 8.22 \\
$\mathrm{R}$ by T & 2,14 & 8.07 \\
Set Form (S) by T & 2,14 & 8.77 \\
Probe Form (P) by S & 1,7 & 26.41 \\
N by S by P & 3,21 & 8.83 \\
T by S by P & 2,14 & 40.98 \\
N by T by S by P & 6,42 & 3.57 \\
R by T by S by P & 2,14 & 9.49 \\
\hline
\end{tabular}

condition had adequate time to encode the presented items. Further, the T functions for the medium and slow conditions were very much like those reported by Cruse and Clifton (1973) in that they were generally linear and markedly steeper than the NT functions. However, the T functions for the fast condition appear to have marked curvature and to be displaced upward relative to the NT functions, contradicting the apparent prediction of the list translation hypothesis. The parameters of straight lines fitted by a least-squares criterion to the mean RTs appear in Table 1.

These impressions are confirmed by statistical analyses performed on the individual Ss' mean RTs. An analysis of variance with set size, set form, probe form, response, 3-day blocks, rate, and $S$ s as factors indicated a number of significant $(\mathrm{p}<.01$ ) effects, listed in Table 2 .

The most notable of these effects are the Probe Form by Set Form and the Probe Form by Set Form by Set Size interactions. An interaction between probe form and set form is equivalent to a contrast between $T$ and NT conditions. These interactions indicate that the RT functions were steeper for the $T$ than for the NT conditions. The Response by Set Size interaction was also significant, indicating that negative RT functions were steeper than positive functions. Such an effect might indicate the operation of a self-terminating memory search strategy.

A number of interactions with rate were significant, including the critical Set Form by Probe Form by Set Size by Rate interaction. Each interaction with rate was further examined by testing the orthogonal contrasts (1) fast vs medium + slow and (2) medium vs slow. The fast vs medium + slow component of each significant interaction with rate (and of the main effect of rate) reached at least the .05 level of significance. However, 
the medium vs slow component of only the main effect of rate and the Set Form by Probe Form by Rate interaction reached the .05 level of significance. These significant effects involving the medium vs slow contrast appear to result from the fact that the difference in mean RT between $\mathrm{T}$ and NT conditions was greater for the medium condition $(37 \mathrm{msec})$ than for the slow condition $(22 \mathrm{msec})$.

The nature of the deviance of the fast condition was examined by performing a separate analysis of variance at each level of the rate factor. In each of these analyses of variance, the effects of set size and the Set Form by Probe Form by Set Size interactions reached the .01 level of significance. In addition, the Set Form by Probe Form and the Response by Set Size interactions reached the .01 level of significance for the fast condition, and the Response by Set Size interaction was significant for the medium condition. For the medium and slow conditions, the linear trend components of the effects and interactions involving set size reached significance, while the deviation from linear trend components did not. Thus, the RT functions for these conditions may be considered to be essentially linear. However, for the fast condition, both the linear trend and the deviation from linear trend components of the set size effect and the Set Form by Probe Form by Set Size interaction reached the .01 level of significance, while only the linear trend component of the Response by Set Size interaction was significant.

The proper description to be given the RT functions in the fast $T$ condition is not yet clear. While the negative response $R T$ function in the fast $T$ condition appears to rise steeply from Set Size 1 to 2 and to be parallel to the fast NT functions from Set Sizes 2 to 4 , the corresponding positive response RT functions appear to rise steeply from Set Size 1 to 3, and only then to become parallel to the NT functions. Common versions of exhaustive serial scanning hypotheses cannot tolerate a difference in form between positive and negative RT functions; therefore, the exact form of the RT functions for the fast condition will be further investigated in Experiment II.

Two final aspects of the data of Experiment I are of interest. First, inspection of Fig. 1 indicates that the zero intercepts of the RT functions for the medium and slow rate $\mathrm{T}$ conditions appear to be lower than the intercepts for the NT conditions. The direction of this effect is just the opposite of that expected if Ss were translating the probe item into the form of the remembered items. However, statistical analyses of the zero intercepts of straight lines fitted to individual Ss' RT data do not confirm this observation. The interactions between probe form and list form in separate analyses of zero intercepts conducted for the medium and slow conditions did not reach significance $[F(1,7)=2.52$ and 3.05 , respectively, $p>.10]$.

Second, the functions relating positive probe $\mathrm{RT}$ to the position of the probe in the presented list were essentially flat for both NT and T data in the medium and slow conditions but had a marked recency effect for the fast NT condition, and primacy and recency effects for the fast $\mathrm{T}$ condition. All but possibly this last finding would be expected on the basis of previous research (Clifton \& Birenbaum, 1970; Cruse \& Clifton, 1973; Corballis, Kirby, \& Miller, 1972). Since comparable, but more precise, serial position curves were obtained in Experiment II, the Experiment I data will not be considered further.

\section{EXPERIMENT II}

The purpose of the second experiment was to determine more precisely the form of the RT functions in the fast presentation conditions. To this end, Ss were given additional practice in the task and were tested only in the fast condition to preclude any inappropriate transfer of strategies from the slower conditions. Further, an attempt was made to reduce the error in the data by making additional observations under each experimental condition, 360 observations per condition in Experiment II as against 144 observations per condition in Experiment I.

\section{Method}

\section{Subjects}

Six Ss were tested for five experimental sessions, following two to five practice sessions and one session during which they were trained on the translation task used. They were paid, as in Experiment I.

\section{Procedure}

Essentially the same procedure was used as in the fast condition of Experiment I, except that sets of one to five (rather than four) items were presented. Twelve blocks of 40 trials were run each day. On each block, each condition defined by the combination of set size (1-5), set form, probe form, and response was tested once.

Due to an error in computer programming, the data for positive probes of Set Size 5/Serial Position 5 were not recorded properly. In addition, approximately $20 \%$ of the positive probes of Set Size 1 were lost. The remaining positive RT data for Set Size 1 and the data for probes of Serial Positions 1-4 for Set Size 5 will be presented, and an attempt will be made to estimate what the Set Size 5 positive RT data would have been if RT to probes of Serial Position 5 had been available.

\section{Results and Discussion}

The means of the Ss' mean correct RTs (with deviant responses eliminated, as in Experiment I), together with the percentages of false alarm and miss errors, are displayed separately for the D-D, L-L, D-L, and L-D conditions (Fig. 2). The data were not collapsed into $T$ and NT conditions because, unlike Experiment I, some major effects involving set form but not probe form were significant. The $95 \%$ confidence interval on these means, estimated from the main analysis of variance, was 
\pm 16.03 msec. $^{1}$ The right-hand panels correspond to the T RT functions and the left-hand to the NT functions. The unfilled circles for Set Size 5 positive probes have been corrected (as described later) for the fact that data for positive probes of the fifth item were missing.

The functions are extremely similar to the functions for the fast condition of Experiment I. Except for the fact that the NT negative probe functions in Experiment II are flatter than the corresponding function for Experiment I (and, thus, more in line with an exhaustive serial scanning process), all major aspects of the Experiment I data can be seen in Fig. 2. In addition, the Set Size 5 data (particularly the corrected data) clearly continue the trends observed in Experiment I for the T RT functions to be parallel at the larger set sizes to the NT functions.

The significant $(p<.01)$ effects of a Set Form by Probe Form by Response by Set Size by Ss analysis of variance performed on the mean RTs (including the uncorrected Set Size 5 data) are listed in Table 3. In general, the effects observed to be significant in Experiment I are significant here also. In addition, there are some effects involving set form (but not probe form) which indicate that lists of letters are dealt with less easily than lists of digits. Further, the Response by Set Size interaction was significant $[\mathbf{F}(4,20)=16.15$, $\mathrm{p}<.011$, as was the Set Form by Probe Form by Response interaction $[F(1,5)=7.65, p<.05]$ and the Set Form by Probe Form by Response by Set Size interaction $[\mathrm{F}(4,20)=4.26, \mathrm{p}<.025]$.

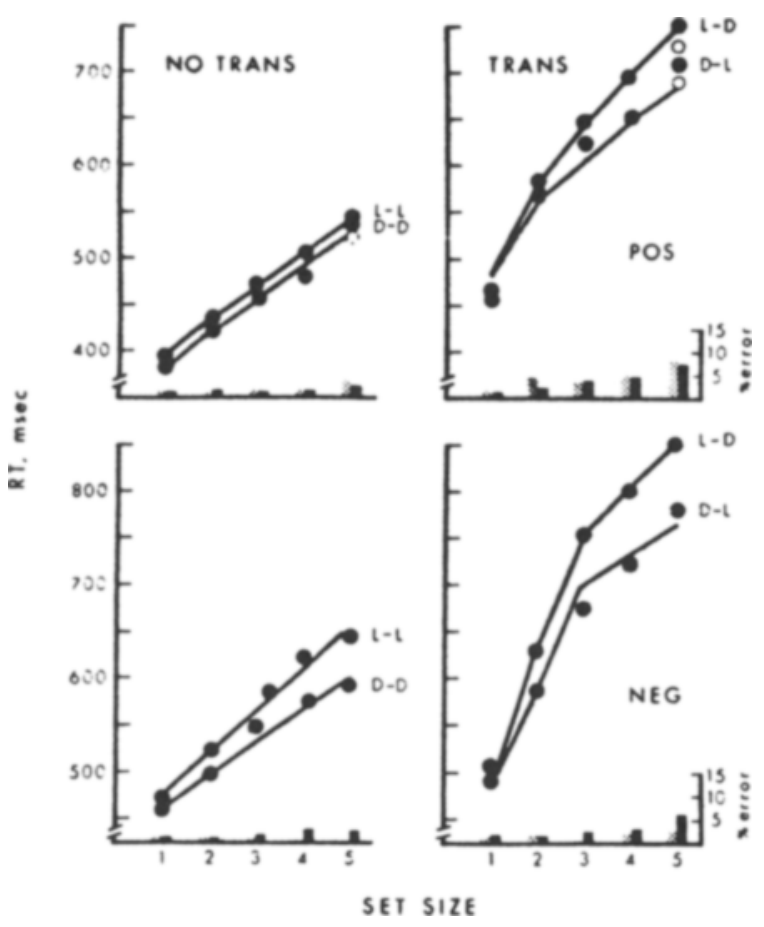

Fig. 2. RT as a function of set size, Experiment II, with \% miss and false alarm errors. Fitted functions are those predicted by the model proposed in the Conclusions section.
Table 3

Significant $(p<.01)$ Effects, Main Analysis: Experiment II

\begin{tabular}{lcc}
\multicolumn{1}{c}{ Effect } & df & F \\
\hline Responses (R) & 1,5 & 37.50 \\
Set Form (S) & 1,5 & 45.34 \\
Set Size (N) & 4,20 & 633.56 \\
R by S & 1,5 & 90.4 \\
S by Probe Form (P) & 1,5 & 50.53 \\
R by N & 4,20 & 16.15 \\
S by N & 4,20 & 25.09 \\
P by N & 4,20 & 4.96 \\
S by P by N & 4,20 & 45.25 \\
\hline
\end{tabular}

The set size effect was partitioned into its linear and deviation from linear trend components. Briefly, both components of each of the significant interactions involving set size in the main analysis were significant $(p<.05)$. Thus, for instance, the significance of both trend components of the List Form by Probe Form by Set Size interaction indicates that $T$ and NT functions differ in steepness and curvature. The NT functions are fair approximations to straight lines (the linear functions fitted to the data account for $98.4 \%$ of the variance among the observed mean RTs). However, the T functions appear to be more curved (only $95 \%$ of the observed variance is accounted for by the linear function). The significance of both trend components of the four-way interaction indicates that the positive and the negative RT functions differ both in slope and in form.

The functions relating positive RT to the position of the probed item in the memorized set (Fig. 3) clearly show a recency effect for the NT data and both recency and primacy effects for the $\mathrm{T}$ data.

These serial position functions were used to estimate the missing value of the mean RT to positive probes of the fifth position of five-item sets. It was assumed that the decrease from Position 4 to Position 5 for five-item sets would be the same as the decrease from Position 3 to Position 4 of four-item sets. This latter decrease was subtracted from the RT to positive probes of the fourth item in five-item sets to estimate the missing Serial Position 5 data. (This procedure probably overestimates these RTs, since the magnitude of the serial position effect seems to increase with set size.) One estimate of the decrease was made for the combined D-D and L-L conditions, and another for the combined D-L and L-D conditions. These estimated points are displayed in Fig. 3. The weighted average of the estimated RT and the obtained RT for the remaining serial positions was then calculated for each condition and taken as the corrected RTs for Set Size 5, displayed in Fig. 2.

\section{CONCLUSIONS}

The translation effect is not indifferent to the rate at which lists are presented, as it should have been if the list translation hypothesis were true. List presentation 


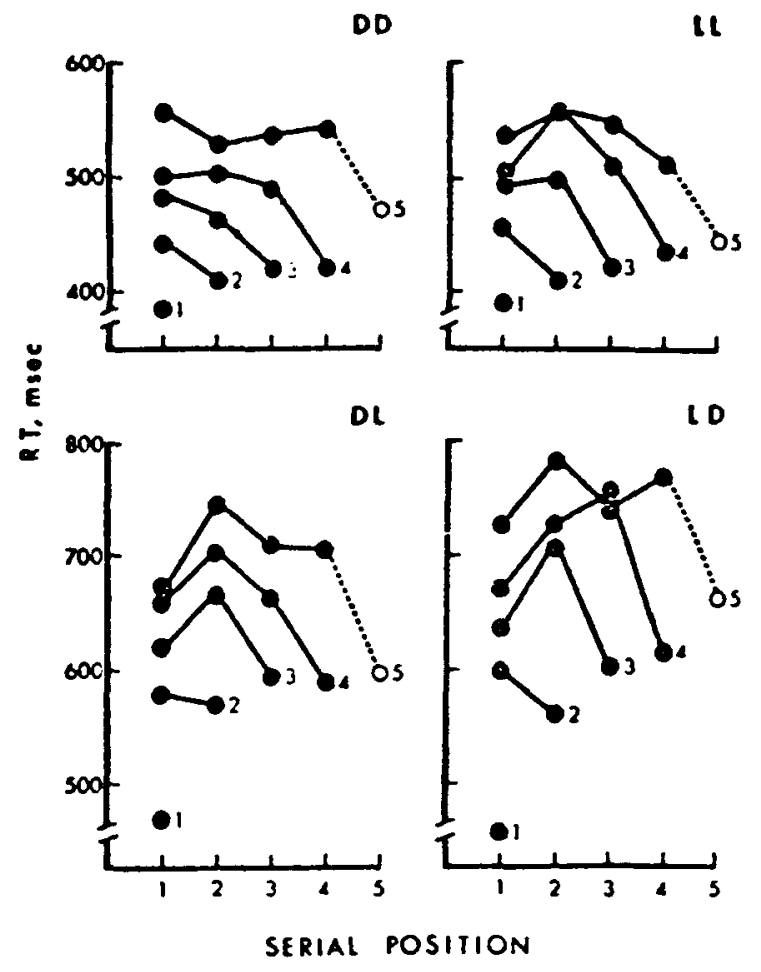

Fig. 3. RT as a function of serial position of positive probes, Experiment II. Serial Position 5 data estimated as described in the text.

rate seemed to affect the form of the translation RT functions.

Other aspects of the data, particularly the fact that positive and negative RT functions for the fast rate condition seemed to have different shapes, create difficulties for simple versions of a serial scanning model. However, a first approximation to a potentially adequate scanning model, which describes the major effects in the data, will be proposed.

Imagine that, when a list of items (e.g., digits) that have translation equivalents (e.g., letters) is presented at a relatively slow rate, $S s$ spend most of their time rehearsing the digits as presented. However, they are able to take a small amount of time to translate the digits into corresponding letters and to rehearse these letters to a minimal degree. (Introspection of some Ss agrees with this suggestion.) The rehearsed digits are held in active memory, while the letters are placed in an inactive but still accessible memory. When a probe item is presented, $S$ identifies it as a digit or as a letter. In the former case, $S$ then begins a relatively rapid process of comparing the item, serially and possibly exhaustively, to the contents of active memory. In the latter case, $S$ compares the item, at a slower rate, to the contents of inactive memory.

In the fast presentation condition, Ss have time to do little more than encode the items as presented and store them in active memory. However, they generally do have time to translate one, and sometimes two, items and place these translated items in inactive memory. (Again, this description accords with the introspections of some Ss.) If a probe is presented in the form of the list items, it is compared at a fast rate against the contents of active memory. If the probe differs in form from the list items, two processes are begun in parallel. The untranslated probe item is compared to the list items that had been translated and placed in inactive memory, at the slow scanning rate characteristic of inactive memory. If a match is found, a "yes" response is initiated. If no match is found and the entire list had been placed in inactive memory, a "no" response is initiated. Simultaneously with this, the probe item is being translated into the form of the items in active memory. If this translation is completed before a response is initiated on the basis of the scan of inactive memory, the translated probe item is compared to the contents of active memory, at the fast rate characteristic of such scans. A response is then initiated on the basis of the result of this comparison. ${ }^{2}$

The model described above was put into quantitative form and fitted to the data for the $T$ conditions of Experiment II. Two parameters were estimated from other available data. The slopes of the NT negative RT functions of Experiment II were used to estimate the rate at which letters and digits are scanned in active memory (45.2 and $34.3 \mathrm{msec} / \mathrm{item}$, respectively), and the slope of the negative response $T$ function from the slow condition of Experiment I was used to estimate the rate at which inactive memory was scanned (79.3 msec/item; no digit-letter difference was apparent in Experiment I). In addition, six parameters were estimated from the 18 mean RT values on the translation RT functions of Experiment II, using STEPIT (Chandler, 1969) to minimize the sum of the squared deviations between predicted and obtained RTs. (The RTs for positive probes of Set Size 5 were disregarded.) The parameters were (1) and (2) base times for positive and negative responses (including all components of RT except scanning and probe translation times), (3) the duration of translating a probe digit into a letter, (4) the duration of translating a probe letter into a digit, (5) the probability that just one digit presented in a list would be translated into a letter and placed into inactive memory (where the complement of this probability is the probability that exactly two digits would be translated into letters), and (6) the probability that just one letter presented in a list would be translated into a digit and placed in inactive memory.

The results of fitting this model to the data of Experiment II, together with the parameter values, are presented in Table 4. Examination of the predicted RTs indicates that they capture the major aspects of the data and fairly closely approximate the obtained RTs. (The lines in Fig. 2 are those predicted by this model.) The root mean square deviation of predicted from obtained values (ignoring positive probes of Set Size 5) was $12.0 \mathrm{msec}$. Further, the obtained parameter values appear reasonable. The estimated translation times of 
215 and $184 \mathrm{msec}$ for letter and digit probes, respectively, are in the region which (if the same translation process operated during the presentation of a list to translate items for placement into inactive memory) would allow Ss to translate just one or two list items at the fast rate used. The fact that the translation time is longer for the letter-digit than for the digit-letter translation is consistent with the fact that the probability of translating two (rather than just one) list digits into letters is higher than the probability of translating two list letters into digits. Further, both of these differences seem consistent with the fact, observed in Experiment II, that the NT functions are higher for letter lists than for digit lists; letter lists seem to be more difficult to deal with generally.

One aspect of the serial position functions of Fig. 3 may be taken to support the model. The primacy and recency effects observed in the translation curves can be understood on the assumption that Ss most frequently translated the first and last items, eliminating the need for the lengthy step of translating the probe when these items were probed.

The model must be taken to be a rough approximation to the truth. Reasonably good fits of a model with six free parameters are not necessarily to be taken as convincing evidence of the accuracy of the model. More than this, though, some possibly unreasonable simplifications were made in the model to avoid additional complexity. For instance, no allowance was made for the possibility that Ss might sometimes translate no list items and sometimes more than two items and that the probability of translating different numbers of items might be a function of set size. Further, estimating the rate of scanning inactive memory from an experiment other than the experiment whose data are being fitted is risky. Finally, there are some notable aberrations in the parameter values and the estimated data points. For instance, the estimated base times for positive and negative responses are much closer together than base times (estimated as zero intercepts of RT functions) generally are in memory experiments.

Nonetheless, one conclusion seems clear. The hypothesis that the items in a remembered list are translated after a probe item of a different form is presented does not adequately account for all data obtained in memory scanning experiments which require translation. A more adequate account claims that Ss translate list items into their alternate forms if possible and scan such translated items more slowly than they scan untranslated items. When such translation of presented items is impossible, Ss resort to translating a probe item to match what they remember of the list. This implies that the recognition-with-translation task cannot be used in the simple fashion suggested by the list translation hypothesis to identify and measure the rate of processes of decoding information from memory. However, interesting questions still remain regarding the nature of the proposed inactive memory and the
Table 4

Observed and Predicted RT, Translation Conditions, With Estimated Parameters: Experiment II

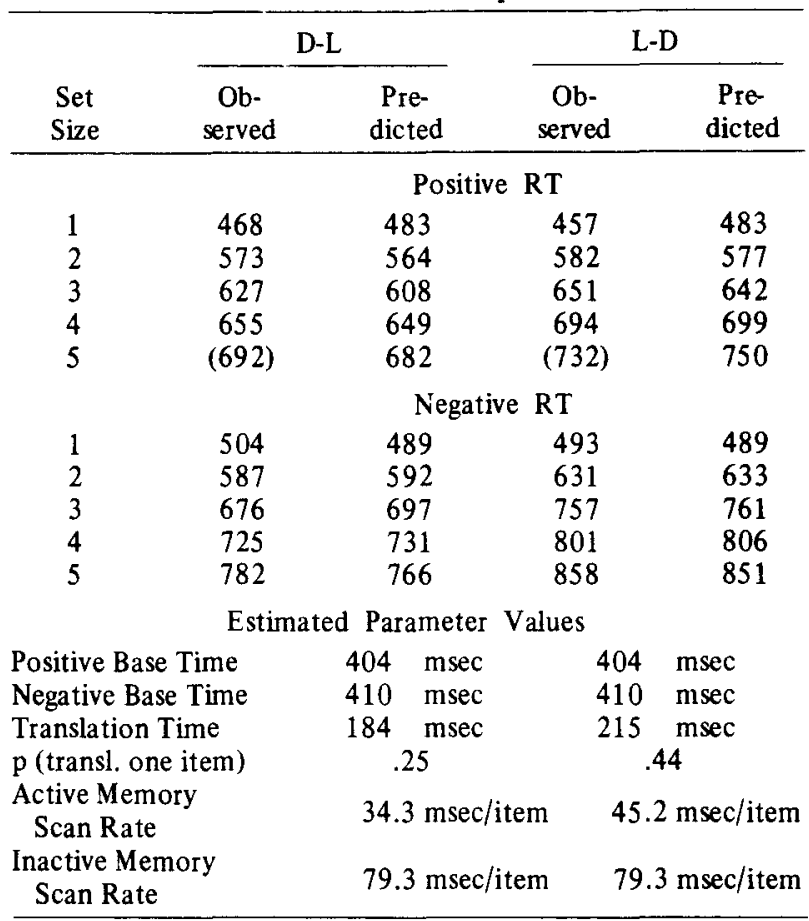

Note-Parenthesized value is corrected for missing data; not included when calculating predicted values. Two estimates of the standard errors of the observed means are available. One is calculated from the highest order error term in the analysis of variance and equals $7.67 \mathrm{msec}$. The other is calculated from the between-S distribution of mean $R T$ for each condition and ranges across conditions from 25.2 to $44.5 \mathrm{msec}$.

processes whereby information is placed into and retrieved from inactive memory.

\section{REFERENCES}

Atkinson, R. C., \& Juola, J. F. Search and decision processes in recognition memory. In D. H. Krantz, R. C. Atkinson, R. D. Luce, and P. Suppes (Eds.), Contemporary developments in mathematical psychology. New York: Academic Press, in press.

Bower, G. H. Organizational factors in memory. Cognitive Psychology, 1970, 1, 18-46.

Bransford, J. D., \& Franks, J. J. The abstraction of linguistic ideas. Cognitive Psychology, 1971, 2, 331-350.

Chandler, J. P. STEPIT: Finds local minima of a smooth function of several parameters. Behavioral Science, 1969, 14, 81-82.

Clifton, C., Jr., \& Birenbaum, S. Effects of serial position and delay of probe in a recognition memory task. Journal of Verbal Learning \& Verbal Behavior, 1971, 10, 528-542.

Corballis, M. C., Kirby, J., \& Miller, A. Acciss to elements of a memorized list. Journal of Experimental Psychology, 1972, 94, 185-190.

Cruse, D., \& Clifton, C., Jr. Recoding strategies and the retrieval of information from memory. Cognitive Psychology, 1973, 4, 157-193.

Mandler, G. Organization and memory. In K. Spence and J. Spence (Eds.), The psychology of learning and motivation. Vol. 1. New York: Academic Press, 1967. Pp. 327-372.

Melton, A. W., \& Martin, E. Coding processes in human memorv 
Washington, D.C: Winston, 1972.

Miller, G. A. The magical number seven, plus or minus two: Some limits on our capacity for processing information. Psychological Review, 1956, 63, 81-97.

Prytulak, L. S. Natural language mediation. Cognitive Psychology, 1971, 2, 1-56.

Sachs, J. S. Recognition memory for syntactic and semantic aspects of connected discourse. Perception \& Psychophysics, $1967,2,437-442$.

Stemberg, S. High-speed scanning in human memory. Science, $1966,153,652-654$.

Stemberg, S. Memory-scanning: Mental processes revealed by reaction-time experiments. American Scientist, 1969, 57, 421-457.

Swanson, J. M., Johnsen, A. M., \& Briggs, G. E. Recoding in a memory search task. Journal of Experimental Psychology, $1972,93,1-9$.

\section{NOTES}

1. Note that, while the confidence interval is not much smaller than that of Experiment I, the means to which it applies result from a finer breakdown of the data than in Experiment $I$.
2. No firm position should be taken yet on the nature of inactive memory; for instance, it is not clear whether inactive and active memories should be taken as structurally different stores or as different levels of memory strength. Two things seem clear, however. First, the strategy described in Sternberg (1969, Experiment 5), in which the apparently slow rate of scanning inactive memory results from the concatenation of the two serial processes of transferring items from inactive to active memory and then scanning active memory, is inappropriate here. If $S s$ in the present fast translation condition had done this, the process of scanning whatever items had been translated during list presentation would leave these translated items in active memory. The originally presented items would then not be in active memory and, thus, not quickly available, if $S$ then had to translate the probe into the form of the presented items and compare it to them. Second, inactive memory should not be identified with the " $E / K$ " store of Atkinson and Juola (in press), in which thoroughly memorized long lists of items are held, since these authors estimated the rate of scanning such long lists to be very fast (under $10 \mathrm{msec} / \mathrm{item}$ ).

(Received for publication March 17, 1973; accepted March 21, 1973.) 